\title{
Non Linear Current Response of a Many-Level Tunneling System: Higher Harmonics Generation
}

\author{
Y.Goldin*and Y.Avishai ${ }^{\dagger}$ \\ Physics Department, Ben Gurion University of the Negev, \\ Beer Sheva, Israel
}

13 May 1996

\begin{abstract}
The fully nonlinear response of a many-level tunneling system to a strong alternating field of high frequency $\omega$ is studied in terms of the Schwinger-Keldysh nonequilibrium Green functions. The nonlinear time dependent tunneling current $I(t)$ is calculated exactly and its resonance structure is elucidated. In particular, it is shown that under certain reasonable conditions on the physical parameters, the Fourier component $I_{n}$ is sharply peaked at $n=\frac{\Delta E}{\hbar \omega}$, where $\Delta E$ is the spacing between two levels. This frequency multiplication results from the highly nonlinear process of $n$ photon absorption (or emission) by the tunneling system. It is also conjectured that this effect (which so far is studied mainly in the context of nonlinear optics) might be experimentally feasible.
\end{abstract}

Submitted to Phys.Rev.B

cond-mat/9605172

*goldin@bgumail.bgu.ac.il

†yshai@bgumail.bgu.ac.il 


\section{Introduction}

The physics of resonance tunneling through a single quantum well is at the center of theoretical and experimental activity for more than two decades. Electron (or hole) confinement between penetrable barriers in a semiconductor enables the investigation of numerous important phenomena such as negative differential resistance, Coulomb blockade, single electron tunneling, single electron pump and many others. The pertinent physics is very attractive both because of its richness and its potential device oriented nature.

At the core of the phenomena of resonance tunneling lies the relatively simple picture of a small cavity connected by tunneling barriers to two particle reservoirs (also termed as leads). The dimensions of the cavity are small enough so that the energy levels inside it are well separated. These levels might be either single particle levels whose spacing is determined solely by geometrical considerations or a few particle levels determined by interactions (as in the Coulomb blockade systems). If there is a difference between the chemical potential of the left lead $\left(\mu_{L}\right)$ and that of the right lead, $\left(\mu_{L}\right)$ a tunneling current occurs between the two leads. If an energy level of electrons in the cavity occurs between $\mu_{L}$ and $\mu_{R}$ this current displays a resonance structure. Evidently, the pertinent physics is time independent, namely, one speaks here of a DC current. Moreover, in most cases, resonance tunneling through a single level can be treated within the formalism of linear response.

Recently, interest is directed toward non linear time dependent transport phenomena in double barrier resonance tunneling systems. Experimentally, investigation of AC current in mesoscopic devices proves to be feasible [1]. (Yet, theoretical analysis of the above experiment remains in the realm of linear response [2, 3, 䜣).

The relevant physics inevitably becomes richer and more difficult to analyze. It touches upon qualitatively new phenomena which depend on how

space and time dependent electronic states interfere. Among the effects which are inherently based on non-linear response one might consider electron pumps [5, 6, 7], lasers [8], photon assisted tunneling [9, 10, 11, 12, 13, 14] and others.

In the present work we concentrate on a relatively new effect, namely, frequency multiplication of current response. As a theoretical model one may consider a double barrier resonance tunneling system containing at least two quantum levels whose spacing $\Delta$ introduces a new energy scale into 
the problem. The system is then subject to a strong monochromatic AC voltage of strength $W$ and frequency $\omega$, which results in a non-linear current response $I(t)$, the main object of our study. The combination of strong AC electric field and level interaction with the electrons in the reservoirs might lead to transitions between these levels which are assisted by many photon emission (or absorption), a highly non-linear effect. It can be analyzed in terms of the Fourier components $I_{n}$ of the current $I(t)$. In linear response, one expects $I_{0}$ and $I_{1}$ to be the only non-zero components. Here, however, higher components may be significant. As we show below, $I_{n}$ as a function of $n$ is peaked at $n=W / \Delta$, namely, there is a resonance when $n$ photons are absorbed or emitted following transition between the two levels. This kind of frequency multiplication is familiar in non-linear optics but, to our best knowledge has not yet been investigated in micro- electronics. We give below some realistic estimates of this effect.

As for the theoretical treatment of the above model, we start from the familiar tunneling Hamiltonian and employ the Schwinger-Keldysh nonequilibrium Green functions, through which the current is calculated in a straightforward way. In order to avoid complications while stressing the important role of two level quantum wells we restrict ourselves in this work to noninteracting particles. Principally, interaction effects can partially be included within screening or Hartree-Fock schemes. As we explain in the next section, the case of a Coulomb blockade system in which the interaction is reflected through the charging energy is qualitatively included in the framework of our approach. The physics of non-linear AC response of a general interacting system in a resonance tunneling device contains new effects which go beyond the scope of the present work.

In the following section the problem is formulated, and the various parts of the pertinent Hamiltonians are defined and justified. Then, in section 3 method of solution in terms of the Keldysh Green function is introduced. In particular, the free particle Green functions are written down and the Dyson equation for the "lesser" Green function is derived. Section 4 is devoted to the discussion of the tunneling current. An exact expression for the current is derived in terms of Keldysh Green functions and the origin of resonances in the non-linear response is explicitly elucidated. Analysis of higher harmonic generation is carried out in section 5, where numerical results are presented and the conditions for obtaining peaks at higher harmonics are discussed. The paper is then concluded with a short summary. In Appendix $A$ there 
is a proof that a pure time dependent potential has no physically observable effect, while some technical point related to the derivation of Dyson equation in the Keldysh formalism is explained in Appendix $B$.

\section{Formulation of the problem}

We consider a structure where a charge carrier has two barriers in its way like the one drawn in fig. 1. The structure is analogous to the Fabry Perot resonator - the motion of a carrier is almost quantized in the central region but it still can escape into the leads. Thus the energy levels of the central region provide resonances for the transmission of the charge carries from one lead to another. Hereafter we will speak about electrons. The same type of structure can be made for holes but degeneracy of the valence band can complicate the calculation. An arbitrary combination of dc- and ac - potentials is applied to the structure (the potential differences are still required to be small compared with the Fermi energies). Our main goal is to calculate the time - dependent currents in the system.

Before doing this let us mention possible experimental realizations. Practically the appropriate structure can be obtained in several ways:

1. By putting several layers of different semiconductors having matching lattices and different gap widths one on top of another [15, 16, 17, 18, 19, 20, 21, 22, 23, 24, 25, 26, 27]. The electrons then move in the direction perpendicular to the layers. As an example, the profile of the conduction band for a particular case of a $G a A s-A l_{x} G a_{1-x} A s$ structure is shown in fig. 11.

2. By application of an external gate potential [10, 11, 28, 29, 30, 31, 32, 33, 34, 35, 36, 37, 40. The electrons then move along the layers but their movement is restricted by the external repulsive potential. The gate is shaped such that the electrons move in one direction and two barriers are formed in their way.

3. By placing an impurity in a tunnel barrier [38, 39, 41].

In order to describe the dynamics of the system we use the tunneling Hamiltonian method [42, 43]. The total Hamiltonian is:

$$
H(t)=H_{f}+H_{a c}(t)+H_{T}
$$


where

$$
H_{f}=\sum_{k} \epsilon_{k} a_{k}^{+} a_{k}+\sum_{n} \epsilon_{n} c_{n}^{+} c_{n}+\sum_{p} \epsilon_{p} b_{p}^{+} b_{p}
$$

is the Hamiltonian of free particles with neither ac-field nor coupling between the leads and the central region. Here $\epsilon_{k}, \epsilon_{n}$ and $\epsilon_{p}$ are free-particle energies in the left lead, the central region and the right lead respectively. The energy is taken with respect to the bottom of the conduction band in the central region. The operators $a_{k}^{+}, a_{k}, b_{p}^{+}, b_{p}, c_{n}^{+}, c_{n}$ are creation and annihilation operators in the leads and in the central region. Furthermore, $k(p)$ are momenta in the direction perpendicular to the layers, $n$ numbers the levels in the central region (hereafter $k(p)$ refer to the left (right) lead, $n, m, n^{\prime}$, $m^{\prime}$ - to the central region), summation over $n$ in (2) expresses the presence of more than one energy level. The third term,

$$
H_{T}=\sum_{k, n}\left(T_{n k}^{L} c_{n}^{+} a_{k}+T_{n k}^{L *} a_{k}^{+} c_{n}\right)+\sum_{p, n}\left(T_{p n}^{R} b_{p}^{+} c_{n}+T_{p n}^{R *} c_{n}^{+} b_{p}\right)
$$

is the part responsible for the tunneling through the barriers, $T_{n k(p)}^{L(R)}$ are transfer matrix elements between the leads and the central region. Finally, the time dependent part is

$$
H_{a c}(t)=W_{L} \sin (\omega t) \cdot \sum_{k} a_{k}^{+} a_{k}+W_{R} \sin (\omega t) \cdot \sum_{p} b_{p}^{+} b_{p}
$$

where $W_{L(R)} \sin (\omega t)$ are potential shifts of the leads with respect to the central region caused by an external ac-potential. dc - potential shifts are included into the energies $\epsilon_{k(n, p)}$. The arrangement in which $W_{L}$ and $W_{R}$ have opposite signs corresponds to an application of an ac-bias as it was done in the experiments 16, 22, 34. The choice $W_{L}=W_{R}$ describes an application of an ac-voltage to the gate electrode superimposed on the central region (see the experiments [10, 11]). We notice here that shifting both leads together (having the potential of the central region fixed) is equivalent to shifting the central region (having the potentials of the leads fixed) since an application of a uniform (time - dependent) potential is not observable even if it is arbitrary strong and arbitrary fast (see Appendix A). The situation $W_{L}=0$, $W_{R} \neq 0$ corresponds to an application of an ac - voltage only to one barrier as it was done in the experiment [10].

Our choice of $H_{a c}$ is based on the following assumptions: 
1. The electrons in the leads respond to an applied field very fast since we deal with the frequencies much less than the plasma frequency. It means that any change of the external potential causes an immediate rearrangement of the electrons. In other words, the internal potential responds very quickly to an external field [2, 14].

2. The concentration of the electrons in the leads is high enough to screen an external field 445, 46, 477. Therefore potential is uniform in the leads and drops in the barriers $99,44,45,47,48,49,50,51,52,53,54,55$.

3. We used a widespread assumption [44, 49, 54, 56] that the probability of direct transitions between the energy levels in the central region due to the ac-field is small and can be neglected.

We do not restrict our consideration to the case of small $W_{L}, W_{R}$ (linear response). They can be arbitrary large. A strong ac-field $\left(W_{L} \gg \omega\right.$ or $W_{R} \gg \omega$ ) leads to a non - linearity. One of its signatures is the generation of current harmonics with frequencies much larger than $\omega$.

The Hamiltonian (11) does not include Coulomb interaction. It is frequently omitted in the treatment of quantum wells $444,45,47,49,50,51,57$, 58, 59, 60, 61, 62, 63, 64, and others]. The number of electrons there is very large so the Coulomb interaction can be treated in the mean - field approximation. In fact it makes concentration - dependent corrections to the one - particle energies $\epsilon_{n}$. It can lead to some changes in the dependence of the current on the bias (such as the appearance of a hysteresis [16, 65, 66, 67]) or, may be, on the magnitude of the ac-field but the physics of the electron - photon interaction remains essentially within the independent particle model. In quantum dots the energy of the Coulomb interaction is much larger than the spacing between one - particle energy levels, level widths and temperature. The dc-bias, the magnitude of the ac-potentials $\left(W_{L}, W_{R}\right)$ as well as the frequency are in general much smaller than the Coulomb interaction energy. Hence, to a good approximation, every tunneling event involves only that electron which happens to have the highest energy. The Coulomb repulsion with the others only provides it with the potential energy needed to penetrate into the leads. Thus they serve as a background. We can forget (at least, qualitatively) about this background in our consideration of the interaction of that electron which provides the current with the ac-field. 
Rigorously speaking, the Hamiltonian (1) is one - dimensional. In QWs there are lateral degrees of freedom provided by the motion along the layers. But at every particular value of the lateral momentum the problem is one dimensional. Even if the dependence of electron energy on lateral momentum is important the total current can found by simple integration over it. The lateral degrees of freedom can be removed by a magnetic field perpendicular to the layers.

\section{Solution in terms of Keldysh Green's func- tions.}

In order to work out the current we first have to analyze the electron propagation. To this end we employ the non-equilibrium Green function technique suggested by Schwinger [68, Kadanoff and Baym [69] and Keldysh [70] (for a review see Ref. [52, 71, 72, 73, 74, 75]). Since no equilibrium is required one can rigorously consider large perturbations and high frequencies drawing the system far from its steady state. The method uses a time variable defined on two sides of the real axis. It is equivalent to introduction two independent Green's functions, one which characterizes the dynamical properties of the particles the other one describes their distribution [70].

It is convenient to use the following two Green functions (the others can be expressed through them):

$$
\begin{aligned}
G_{i, j}^{r}\left(t_{1}, t\right) & =-i \theta\left(t_{1}-t\right)\left\langle\varphi_{i}\left(t_{1}\right) \varphi_{j}^{\dagger}(t)+\varphi_{j}^{\dagger}(t) \varphi_{i}\left(t_{1}\right)\right\rangle \\
G_{i, j}^{<}\left(t_{1}, t\right) & =i\left\langle\varphi_{j}^{\dagger}(t) \varphi_{i}\left(t_{1}\right)\right\rangle
\end{aligned}
$$

where $\varphi_{i}\left(t_{1}\right)\left(\varphi_{j}^{\dagger}(t)\right)$ are operators in the Heisenberg picture representing $a_{k}\left(t_{1}\right), c_{n}\left(t_{1}\right), b_{p}\left(t_{1}\right)\left(a_{k}^{\dagger}(t), c_{n}^{\dagger}(t), b_{p}^{\dagger}(t)\right)$ in correspondence with what values the indexes $i$ and $j$ take $(k, n$ or $p)$. The first one is the usual retarded Green function. The advanced Green function $\left(G_{i j}^{a}\right)$ is its Hermitian conjugate that is: $G_{i, j}^{a}\left(t_{1}, t\right)=\left[G_{j i}^{r}\left(t, t_{1}\right)\right]^{*}$. The other one contains information about the distribution of electrons and their correlations. At $t_{1}=t$ it gives the one - particle density matrix $(\rho)[71]: G_{i j}^{<}(t, t)=i N \rho_{i j}(t)$, where $N$ is the number of particles in the system. The advantage of the method is that it 
treats $G^{r}$ and $G^{<}$in a unified manner. Both of them can be found through the Dyson's equation [73]:

$$
\begin{aligned}
G^{r} & =g^{r}\left[1+\Sigma^{r} G^{r}\right] \\
G^{<} & =\left[1+G^{r} \Sigma^{r}\right] g^{<}\left[1+\Sigma^{a} G^{a}\right]+G^{r} \Sigma^{<} G^{a}
\end{aligned}
$$

Here multiplication implies summation (or integration) over space variables and integration over time, $g^{r}$ and $g^{<}$are Green functions defined as (5) but for an unperturbed Hamiltonian, $\Sigma^{r}, \Sigma^{a}$ and $\Sigma^{<}$are proper irreducible self energies.

An application of the formalism to tunneling systems is especially powerful if one chooses $H_{T}$ as a perturbation [76]). Then then self-energies assume a very simple form:

$$
\begin{aligned}
& \Sigma_{k k^{\prime}}^{r(a)}=\Sigma_{p p^{\prime}}^{r(a)}=\Sigma_{k p}^{r(a)}=\Sigma_{p k}^{r(a)}=0 \\
& \Sigma_{n k}^{r}=\Sigma_{n k}^{a}=T_{n k}^{L} \\
& \Sigma_{k n}^{r}=\Sigma_{k n}^{a}=T_{k n}^{L}=T_{n k}^{L *} \\
& \Sigma_{n p}^{r}=\Sigma_{n p}^{a}=T_{n p}^{R} \\
& \Sigma_{p n}^{r}=\Sigma_{p n}^{a}=T_{p n}^{R}=T_{n p}^{R *} \\
& \Sigma^{<} \equiv{ }^{<}
\end{aligned}
$$

This is due to the fact that any vertex caused by $H_{T}$ has only two entries. The only diagram contributing to an irreducible self - energy is the simplest one drawn in fig. 2 $2 \mathrm{a}$. The next one drawn in fig. 2 $\mathrm{b}$ is already reducible.

In the next section we will show that tunneling currents can be expressed solely in terms of the Green functions in the central region. To find them we iterate (6) and get:

$$
\begin{aligned}
G_{n m}^{r}\left(t_{1}, t\right) & =g_{n m}^{r}\left(t_{1}, t\right)+ \\
& +\sum_{n^{\prime}} \int_{-\infty}^{+\infty} \int_{-\infty}^{+\infty} d t_{2} d t_{3} g_{n n}^{r}\left(t_{1}, t_{2}\right) X_{n n^{\prime}}\left(t_{2}, t_{3}\right) G_{n^{\prime} m}^{r}\left(t_{3}, t\right) \\
& X_{n n^{\prime}}\left(t_{2}, t_{3}\right) \equiv X_{n n^{\prime}}^{L}\left(t_{2}, t_{3}\right)+X_{n n^{\prime}}^{R}\left(t_{2}, t_{3}\right) \\
& X_{n n^{\prime}}^{L}\left(t_{2}, t_{3}\right) \equiv \sum_{k} T_{n k}^{L} g_{k k}^{r}\left(t_{2}, t_{3}\right) T_{k n^{\prime}}^{L} \\
& X_{n n^{\prime}}^{R}\left(t_{2}, t_{3}\right) \equiv \sum_{p} T_{n p}^{R} g_{p p}^{r}\left(t_{2}, t_{3}\right) T_{p n^{\prime}}^{R}
\end{aligned}
$$


Equation (7)-(8) establish an algorithm for calculating $G_{n m}^{<}$without an iteration but nevertheless it contains eleven terms on the right hand side. We showed (see Appendix B) that only two of them remain after a long enough period of time has passed from the moment the tunneling was switched on:

$$
G_{n m}^{<}\left(t_{1}, t\right)=\sum_{n^{\prime} m^{\prime}} \int_{-\infty}^{+\infty} \int_{-\infty}^{+\infty} d t_{2} d t_{3} G_{n n^{\prime}}^{r}\left(t_{1}, t_{2}\right) Y_{n^{\prime} m^{\prime}}\left(t_{2}, t_{3}\right) G_{m^{\prime} m}^{a}\left(t_{3}, t\right),
$$

where

$$
\begin{aligned}
Y_{n^{\prime} m^{\prime}}\left(t_{2}, t_{3}\right) & \equiv Y_{n^{\prime} m^{\prime}}^{L}\left(t_{2}, t_{3}\right)+Y_{n^{\prime} m^{\prime}}^{R}\left(t_{2}, t_{3}\right) \\
Y_{n^{\prime} m^{\prime}}^{L}\left(t_{2}, t_{3}\right) & \equiv \sum_{k} T_{n^{\prime} k}^{L} g_{k k}^{<}\left(t_{2}, t_{3}\right) T_{k m^{\prime}}^{L} \\
Y_{n^{\prime} m^{\prime}}^{R}\left(t_{2}, t_{3}\right) & \equiv \sum_{p} T_{n^{\prime} p}^{R} g_{p p}^{<}\left(t_{2}, t_{3}\right) T_{p m^{\prime}}^{R}
\end{aligned}
$$

We notice that $G_{n m}^{<}$does not depend on the initial distribution of electrons in the central region (because $g_{n m}^{<}$do not appear in formula (11)). It depends only on their distributions in the leads through $g_{k k}^{<}$and $g_{p p}^{<}$.

It is easy to show (see Appendix A) that even under a time-dependent potential of an arbitrary large amplitude and an arbitrary high frequency the Green functions for electrons in isolated leads are given by "adiabatic - like" expressions:

$$
\begin{aligned}
g_{k k^{\prime}}^{r}\left(t_{2}, t_{3}\right) & =-i \delta_{k k^{\prime}} \Theta\left(t_{2}-t_{3}\right) e^{-i \int_{t_{3}}^{t_{2}}\left[H_{f}+H_{a c}(t)\right]_{k k} d t}= \\
& =-i \delta_{k k^{\prime}} \Theta\left(t_{2}-t_{3}\right) e^{-i \epsilon_{k}\left(t_{2}-t_{3}\right)+i \frac{W_{L}}{\omega}\left(\cos \left(\omega t_{2}\right)-\cos \left(\omega t_{3}\right)\right)} \\
g_{k k^{\prime}}^{<}\left(t_{2}, t_{3}\right) & =i \delta_{k k^{\prime}} f_{L}\left(\epsilon_{k}\right) e^{-i \int_{t_{3}}^{t_{2}}\left[H_{f}+H_{a c}(t)\right]_{k k} d t}= \\
& =i \delta_{k k^{\prime}} f_{L}\left(\epsilon_{k}\right) e^{-i \epsilon_{k}\left(t_{2}-t_{3}\right)+i \frac{W_{L}}{\omega}\left(\cos \left(\omega t_{2}\right)-\cos \left(\omega t_{3}\right)\right)}
\end{aligned}
$$

provided that the time - dependent perturbing potential $\left(H_{a c}\right)$ is uniform in every lead. Here $[\ldots]_{k k}$ denotes matrix element, $f_{L}\left(\epsilon_{k}\right)=\frac{1}{e^{\frac{\epsilon_{k}-\mu_{L}}{k \Theta}}+1}$ is the Fermi function for the left lead, $\mu_{L}$ is its chemical potential, $\Theta$ is the temperature. Formulas for $g_{p p^{\prime}}^{r}, g_{p p^{\prime}}^{<}$can be obtained from (13) by replacements $k, k^{\prime} \rightarrow p, p^{\prime}, W_{L} \rightarrow W_{R}, \mu_{L} \rightarrow \mu_{R}$.

Now we substitute (13) into (10) and (12) and replace the sum over $k, p$ by an integral over energy. Further, we employ the "wide - band approximation" [77, 78] assuming the elastic coupling to the leads

$$
\Gamma_{n n^{\prime}}^{L} \equiv 2 \pi \rho\left(\epsilon_{k}\right) T_{n k}^{L} T_{k n^{\prime}}^{L} \quad \text { and } \quad \Gamma_{n n^{\prime}}^{R} \equiv 2 \pi \rho\left(\epsilon_{p}\right) T_{n p}^{R} T_{p n^{\prime}}^{R}
$$


to be energy independent (i.e. they do not depend on $k, \epsilon_{k}$ ) within the band. The band widths and the Fermi energies are large compare to $W_{L}, W_{R}$. Thus (10) and (12) take a rather simple form:

$$
\begin{aligned}
X_{n n^{\prime}}\left(t_{1}, t_{2}\right) & =-\frac{i}{2} \Gamma_{n n^{\prime}} \delta\left(t_{1}-t_{2}\right) \\
Y_{n^{\prime} m^{\prime}}\left(t_{1}, t_{2}\right) & =i \Gamma_{n^{\prime} m^{\prime}}^{L} \int_{V_{L}}^{+\infty} \frac{d \epsilon_{k}}{2 \pi} f_{L}\left(\epsilon_{k}\right) e^{-i \epsilon_{k}\left(t_{1}-t_{2}\right)+i \frac{W_{L}}{\omega}\left(\cos \left(\omega t_{1}\right)-\cos \left(\omega t_{2}\right)\right)} \\
& +i \Gamma_{n^{\prime} m^{\prime}}^{R} \int_{V_{R}}^{+\infty} \frac{d \epsilon_{p}}{2 \pi} f_{R}\left(\epsilon_{p}\right) e^{-i \epsilon_{p}\left(t_{1}-t_{2}\right)+i \frac{W_{R}}{\omega}\left(\cos \left(\omega t_{1}\right)-\cos \left(\omega t_{2}\right)\right)}
\end{aligned}
$$

Hereafter $V_{L}\left(V_{R}\right)$ are energies of the conduction band bottoms in the left (right) lead, $\Gamma_{n n^{\prime}} \equiv \Gamma_{n n^{\prime}}^{L}+\Gamma_{n n^{\prime}}^{R}$. We can not put $V_{L}, V_{R} \rightarrow-\infty$ in the formula (16) because it would lead to a divergence in the ac-current.

We want to emphasize that substituting (15) into (9) yields a time independent equation. The problem then reduces to the task of finding the usual retarded Green function for a time - independent potential well with finite barriers. This is due to the energy independence of $\Gamma_{n n^{\prime}}^{L(R)}$. The Fourier transform of the equation is:

$$
G_{n m}^{r}(\epsilon)+\frac{i}{2} g_{n n}^{r}(\epsilon) \sum_{n^{\prime}} \Gamma_{n n^{\prime}} G_{n^{\prime} m}^{r}(\epsilon)=g_{n m}^{r}(\epsilon)
$$

A rigorous solution for a two - level system shows that the mixing terms $G_{n m}^{r}, n \neq m$ give a contribution to the current of the order of $\Gamma /\left|\epsilon_{n}-\epsilon_{m}\right|$. We assume $\Gamma \ll\left|\epsilon_{n}-\epsilon_{m}\right|$ and drop them out hereafter. With the same accuracy $G_{n n}^{r}$ are given by:

$$
G_{n n}^{r}(\epsilon)=\frac{1}{\epsilon-\epsilon_{n}+i \frac{\Gamma_{n}}{2}}
$$

where $\Gamma_{n} \equiv \Gamma_{n n}+\Gamma_{n}^{\text {in }}, \Gamma_{n}^{\text {in }}$ are intrinsic level widths due to inelastic interactions, leakage of electrons into lateral directions etc. This is equivalent to the widely - used assumption that the quasilevels of a potential well with finite barriers have complex energies $\epsilon_{n}-i \frac{\Gamma_{n}}{2}$. We see that the main contribution of tunneling in the dynamical properties is level broadening. 
Substituting (16) into (11) gives an exact expression for $G^{<}$as soon as we know $G^{r}$ :

$$
\begin{array}{r}
G_{n m}^{<}\left(t_{1}, t\right)=\int_{V_{L}}^{+\infty} \frac{d \epsilon_{k}}{2 \pi} f_{L}\left(\epsilon_{k}\right) e^{-i \epsilon_{k}\left(t_{1}-t\right)} \sum_{s, q=-\infty}^{\infty} i^{s-q+1} J_{s}\left(\frac{W_{L}}{\omega}\right) J_{q}\left(\frac{W_{L}}{\omega}\right) e^{-i s \omega t_{1}+i q \omega t} . \\
\cdot \sum_{n^{\prime} m^{\prime}} \Gamma_{n^{\prime} m^{\prime}}^{L} G_{n n^{\prime}}^{r}\left(\epsilon_{k}+s \omega\right) G_{m m^{\prime}}^{r *}\left(\epsilon_{k}+q \omega\right)+(19) \\
+\int_{V_{R}}^{+\infty} \frac{d \epsilon_{p}}{2 \pi} f_{R}\left(\epsilon_{p}\right) e^{-i \epsilon_{k}\left(t_{1}-t\right)} \sum_{s, q=-\infty}^{\infty} i^{s-q+1} J_{s}\left(\frac{W_{R}}{\omega}\right) J_{q}\left(\frac{W_{R}}{\omega}\right) e^{-i s \omega t_{1}+i q \omega t} . \\
\cdot \sum_{n^{\prime} m^{\prime}} \Gamma_{n^{\prime} m^{\prime}}^{R} G_{n n^{\prime}}^{r}\left(\epsilon_{p}+s \omega\right) G_{m m^{\prime}}^{r *}\left(\epsilon_{p}+q \omega\right)
\end{array}
$$

We notice that the transition amplitudes $G_{n m}^{<}, n \neq m$ are important as we show in the next section.

This difference between $G_{n m}^{r}$ and $G_{n m}^{<}, n \neq m$ arises from the difference between $X$ and $Y$ in the formulas (15), (16). The quantity $X$ has such a simple form because $f\left(\epsilon_{k(p)}\right)$ does not appear in $g_{k k}^{r}, g_{p p}^{r}$ so the summation over $k(p)$ (equivalent to integration over $\epsilon_{k}\left(\epsilon_{p}\right)$ ) in formula (10) gives a $\delta$ function $\delta\left(t_{1}-t_{2}\right)$ if the band (i.e the range of $\left.\epsilon_{k(p)}\right)$ is wide. The occurrence of $f\left(\epsilon_{k(p)}\right)$ in $g_{k k}^{<}\left(g_{p p}^{<}\right)$leads to an upper limit of integration in (19) around $\mu_{L(R)}$. If $\mu_{L(R)}$ is large enough the $\delta\left(t_{1}-t_{2}\right)$ is recovered. Then the whole solution becomes invariant under time translation and the transition amplitudes $G_{n m}^{<}$, $n \neq m$ are small, of the order of $\Gamma /\left|\epsilon_{n}-\epsilon_{m}\right|$.

\section{The current}

A double barrier structure is integrated within a circuit. The measured current is determined by its influence on the circuit. If the barriers are modeled as capacitive - resistant elements [79] the currents in the leads are given by [12]:

$$
\begin{aligned}
I_{L}(t) & =\frac{C_{R}+C_{g}}{C} C_{L} \omega W_{L} \cos (\omega t)-\frac{C_{L}}{C} C_{R} \omega W_{R} \cos (\omega t)+ \\
& +\frac{C_{R}+C_{g}}{C} I_{L}^{T}(t)-\frac{C_{L}}{C} I_{R}^{T}(t)
\end{aligned}
$$

where $I_{L}$ is the total current in the left lead $I_{L}^{T}(t)$ and $I_{R}^{T}(t)$ are tunneling currents through the left and right barriers respectively, $C_{L(R)}$ and $C_{g}$ are 
capacitances of the central region relative to the left (right) lead and a gate electrode (or another background), $C \equiv C_{L}+C_{R}+C_{g}$. The current in the right lead is obtained by replacing $L$ by $R$. The first two terms describe the contribution of capacitive currents (due to the presence of accumulation and depletion layers). They have the frequency of the input ac - voltage. But the tunneling currents contain also higher harmonics as we show below.

The tunneling current from the left lead into the central region is defined by the change in the number of electrons in that lead:

$$
\begin{aligned}
I_{L}^{T}(t)=e\left\langle\frac{d \hat{N}_{L}}{d t}\right\rangle & =-\frac{i e}{\hbar} \sum_{k m}\left(T_{m k}^{L}\left\langle c_{m}^{+} a_{k}\right\rangle-T_{m k}^{L *}\left\langle a_{k}^{+} c_{m}\right\rangle\right) \\
& =-\frac{2 e}{\hbar} \operatorname{Re}\left[\sum_{k m} T_{m k}^{L} G_{k m}^{<}(t, t)\right]
\end{aligned}
$$

where $\hat{N}_{L} \equiv \sum_{k} a_{k}^{+} a_{k}$. The tunneling current from the right lead into the central region $\left(I_{R}^{T}\right)$ is obtained from the above expression by a change $L \rightarrow R$, $k \rightarrow p, a_{k} \rightarrow b_{p}$.

Using Dyson's equation for $G_{k n}^{<}$allows one to express the tunneling currents through Green functions in the central region (which we found in the previous section):

$I_{L(R)}^{T}(t)=-\frac{2 e}{\hbar} \operatorname{Re}\left\{\sum_{n m} \int_{-\infty}^{+\infty} d t_{1}\left[X_{m n}^{L(R)}\left(t, t_{1}\right) G_{n m}^{<}\left(t_{1}, t\right)+Y_{m n}^{L(R)}\left(t, t_{1}\right) G_{n m}^{a}\left(t_{1}, t\right)\right]\right\}$

Under the assumption of energy independent coupling to the leads which we used in the previous section (see $(\sqrt[14]{14})$ ) we can use the formulas (15), (16), (18) and (19). Dropping out the terms that are always small of the order of $\Gamma /\left|\epsilon_{j}-\epsilon_{j^{\prime}}\right|$ in comparison with the others we obtain after some algebra:

$$
\begin{aligned}
I_{L}^{T}(t)= & \frac{1}{2} I_{L}^{0}+\sum_{n=1}^{+\infty}\left|I_{L}^{n}\right| \cos \left(n \omega t+\phi_{L}^{n}\right), \text { where } \phi_{L}^{n}=\arg I_{L}^{n} \\
I_{L}^{n}= & \frac{2 e}{\hbar} \sum_{j}\left[A_{j}(n)+A_{j}^{*}(-n)+B_{j}(n)+B_{j}^{*}(-n)\right] \\
A_{j}(n)= & i^{-n}\left[i \Gamma_{j j}^{L}+\sum_{j^{\prime}} \frac{\left|\Gamma_{j j^{\prime}}^{L}\right|^{2}}{\epsilon_{j^{\prime}}-\epsilon_{j}-n \omega+i \frac{\Gamma_{j}+\Gamma_{j^{\prime}}}{2}}\right] . \\
& \cdot \sum_{s=-\infty}^{+\infty} J_{s}\left(\frac{W_{L}}{\omega}\right) J_{s+n}\left(\frac{W_{L}}{\omega}\right) F\left(\frac{\epsilon_{j}-\mu_{L}}{\omega}-s, \frac{\mu_{L}-V_{L}}{\omega}, \frac{\Gamma_{j}}{\omega}, \frac{k \Theta}{\omega}\right)
\end{aligned}
$$




$$
\begin{gathered}
B_{j}(n)=i^{-n} \sum_{j^{\prime}} \frac{\Gamma_{j j^{\prime}}^{L} \Gamma_{j^{\prime} j}^{R}}{\epsilon_{j^{\prime}}-\epsilon_{j}-n \omega+i \frac{\Gamma_{j}+\Gamma_{j^{\prime}}}{2}} \cdot \\
\cdot \sum_{s=-\infty}^{+\infty} J_{s}\left(\frac{W_{R}}{\omega}\right) J_{s+n}\left(\frac{W_{R}}{\omega}\right) F\left(\frac{\epsilon_{j}-\mu_{R}}{\omega}-s, \frac{\mu_{R}-V_{R}}{\omega}, \frac{\Gamma_{j}}{\omega}, \frac{k \Theta}{\omega}\right) \\
F\left(\frac{\epsilon_{j}-\mu}{\omega}-s, \frac{\mu-V}{\omega}, \frac{\Gamma_{j}}{\omega}, \frac{k \Theta}{\omega}\right)=\frac{1}{2 \pi} \int_{V}^{+\infty} \frac{f(\epsilon) d \epsilon}{\epsilon+s \omega-\epsilon_{j}+i \frac{\Gamma_{j}}{2}}, f(\epsilon)=\frac{1}{e^{\frac{\epsilon-\mu}{k \Theta}}+1}
\end{gathered}
$$

Analogous expressions hold for $I_{R}^{T}(t)$ as well. For the rest of our discussion we choose to study $I_{L}^{T}$. We point out that the current consists of many harmonics with the frequencies $n \omega$. The sums over $j, j^{\prime}$ express summation over all energy levels in the central region. We notice that the terms $A_{j}(n)$ and $B_{j}(n)$ are quite similar in structure. The first one includes the currents due to direct photon - assisted transitions between the left lead and the central region and due to the effect of the left lead on the population of the central region. The second one describes the current through the left barrier due to the influence of the right lead on the electrons in the central region. The main difference between them (beside a few replacements of $\mathrm{L}$ into $\mathrm{R}$ ) is that the first term in the brackets in $A$ (i.e. $i \Gamma_{j j}^{L}$ ) is absent in $B_{j}(n)$. At zero temperature the integral in the expression for $F$ can be easily computed:

$$
F\left(\frac{\epsilon_{j}-\mu}{\omega}-s, \frac{\mu-V}{\omega}, \frac{\Gamma_{j}}{\omega}, 0\right)=\frac{1}{2 \pi} \ln \frac{\epsilon_{j}-s \omega-\mu-i \frac{\Gamma_{j}}{2}}{\epsilon_{j}-s \omega-V-i \frac{\Gamma_{j}}{2}}
$$

The rest of our calculation is done at zero temperature.

The term in A containing $\Gamma_{j j}$ gives an additive contribution of different energy levels to the current. The same is true for the terms of the sums over $j^{\prime}\left(\right.$ in $A_{j}(n)$ and $\left.B_{j}(n)\right)$ with $j^{\prime}=j$. The other terms in these sums are caused by the presence of different energy levels together. Every one is caused by a certain pair of energy levels. It is obvious that they have a resonance nature. The $j^{\prime}$-th term can be large when $\left|\epsilon_{j^{\prime}}-\epsilon_{j}-n \omega\right|<\left(\Gamma_{j^{\prime}}+\Gamma_{j}\right) / 2$. Thus it can give large ac-current at $n_{\text {res }} \approx\left|\epsilon_{j^{\prime}}-\epsilon_{j}\right| / \omega$ (under condition $W>\left|\epsilon_{j^{\prime}}-\epsilon_{j}\right|$ ). If $\omega \ll\left|\epsilon_{j^{\prime}}-\epsilon_{j}\right|$ one has $n_{\text {res }} \gg 1$, i.e. the frequency in the output is much larger than in the input. We notice that this term generates the $n_{\text {res }}$-th harmonic exclusively. The others are small of the order of $\frac{\Gamma_{j}+\Gamma_{j^{\prime}}}{2 \omega\left(n-n_{\mathrm{res}}\right)}$ (we suppose high frequency $\Gamma / \omega \ll 1)$. 
The resonance is caused by many - photon absorption due to the strength $(W \gg \omega)$ of the ac-field. The formula (21) expresses the current at the moment of time $t$ through the probability to create an electron in the state $m$ annihilating it in the state $k$ as it is drawn in fig. 3a. To do that it is necessary to have an electron in the state $k$ and a hole in the state $m$. The formula (22) shows that it is provided by creation of an electron in the state $k$ and a hole in the state $n$ at an earlier moment of time $t_{1}, t_{1} \leq t$ (see fig. $3 \mathrm{~b}$ ). In the presence of a strong ac - field the process in which $n \neq m$ should be considered. The hole then has to propagate from $n$ to $m$. The formula (11) expresses the transition from $n$ to $m$ through the kind of process drawn in fig. $3 \mathrm{c}$ for $t_{3}<t_{2}$. An electron is transfered at the moment $t_{3}, t_{3} \leq t$ from the state $m$ to the state $p$ in a lead leaving a hole in the state $m$. At the moment $t_{2}, t_{2} \leq t_{1}$ the electron tunnels to the state $n$. Eventually an electron is found in the state $n$ at $t_{1}$ and a hole is found in the state $m$ at $t$. In the absence of a time - dependent potential this process is impossible because the transitions from $m$ to $p$ and from $p$ to $n$ have to conserve energy (after integration over $\left.t_{2}, t_{3}\right)$. Thus $m$ has to be equal to $n$. $H_{a c}(t)$ leads to a multiplication the wave function of the state $p$ by the phase factor $e^{i \frac{W_{R}}{\omega} \cos (\omega t)}$ (see Appendix A and formula (13) in the main text). In the energy representation the wave function becomes dispersed over all energies $\epsilon_{p} \pm s \omega, s$ integer with the weight $J_{s}\left(\frac{W_{R}}{\omega}\right)$. In other words the spectral function of the state $p$ has extra peaks with energies $\epsilon_{p} \pm s \omega$. Their magnitudes are proportional to $J_{s}^{2}\left(\frac{W_{R}}{\omega}\right)$ (see [9]). Thus it is possible to have transitions from $m$ to $p$ and from $p$ to $n$ when

$$
\epsilon_{m}-\epsilon_{p}=q \omega, \quad \epsilon_{n}-\epsilon_{p}=s \omega
$$

This is the origin of $J_{s}$ and $J_{q}$ in the formulas (19), (23). These transitions are accompanied by emission (absorption) of $q(s)$ photons. The total number of absorbed photons is $s-q=n$. When ac-field is strong $\left(W_{R} \gg \omega\right)$ $J_{s}\left(\frac{W_{R}}{\omega}\right)$ and $J_{q}\left(\frac{W_{R}}{\omega}\right)$ can be large for $s, q \gg 1$. This leads to a possibility of many - photon absorption (emission). The resonance conditions (24) give $n_{\text {res }}=s-q=\frac{\epsilon_{m}-\epsilon_{n}}{\omega}$. It is easy to show that, in fact, $W_{R}>n_{\text {res }} \omega$ is required to obtain large $n_{\text {res }}$-th harmonic. The physical content of fig. 3 $3 \mathrm{c}$ expresses the contribution of $Y_{n m}^{R}\left(t_{2}, t_{3}\right)$ into (11). The same process but through the left lead expresses the contribution of $Y_{n m}^{L}\left(t_{2}, t_{3}\right)$. At $t_{2}<t_{3}$ the picture is very similar. The process just starts from a transfer of an electron from $p$ to $n$ at $t_{2}$. Then the hole tunnels from $p$ to $m$ at $t_{3}$. 
If we do not assume that the lead energy bands are wide (in particular that $\left|V_{L(R)}\right|$ is large), then the only change at $W_{L}=0$ (which is required in order to observe the high frequency resonance as we show in the next section) is that now $G_{n m}^{r(a)}, n \neq m$ is not negligible. These non-diagonal elements of the retarded Green functions could make a positive or negative contribution to the process drawn in fig. 3 $\mathrm{c}$. Yet, we argue that the resonance would persist also in that case.

Every pair of energy levels in the central region gives an independent contribution to the current (23). In the next section we consider one pair of energy levels and explore the dependence of the current it produces on the parameters of the system.

\section{High harmonics generation.}

In this section we consider a strong ac - field $\left(W_{L} \gg \omega\right.$ or $\left.W_{R} \gg \omega\right)$ and show the dependence of the current on the parameters of the system. Since every pair of energy levels gives a separate contribution to the current it is enough to consider only one pair.

Generally a strong ac - field leads to generation of high harmonics. In fig. The plot the amplitude of the harmonics $\left(\left|I_{L}^{n}\right|\right)$ versus their number $n$ for (as an example) $W_{L}=W_{R}=30, \epsilon_{1}=11.5, \epsilon_{2}=30.5, \mu_{R}=20, \mu_{L}=5$, $V_{R}=V_{L}=-50, \Gamma_{11}^{L(R)}=\Gamma_{22}^{L(R)}=\Gamma_{12}^{L}=-\Gamma_{12}^{R}=0.05, \omega=1$. We did not show the dc - component because its magnitude is much larger. We did not show the first harmonic (whose amplitude is also a few times larger than that of the others) since it is anyhow only a part of the current response with the frequency $\omega$. The other part is given by the capacitive currents (first terms in the formula (20). The main contribution to the broadened spectrum plotted in fig. 4 is due to the direct photon - assisted transitions from the left lead to the central region and back described by the first term in the braces in $A_{j}(n)$ (see formula (23)). The terms with $j \neq j^{\prime}$ arising from the presence of two or more levels together can lead to generation of one solitary (and high) harmonic (see fig.5). In the rest of this section we describe the conditions required to observe one high harmonic alone and show its dependence on the parameters of the system.

Since the first term in $A_{j}(n)$ generates many harmonics it must be eliminated. The easiest way to do it is to apply ac - voltage only to the right 
barrier: $W_{L}=0$ (in fact it is enough to have $W_{L} \ll\left|\epsilon_{2}-\epsilon_{1}\right|$ ). Then $A_{j}(n)=0, n \neq 0$ because $J_{s}\left(\frac{W_{L}}{\omega}\right)=J_{s}(0)=0, s \neq 0$.

In fig. 5 we plotted the amplitude of the harmonics $\left(\left|I_{L}^{n}\right|\right)$ versus their number $n$ for $W_{L}=0, W_{R}=30, \epsilon_{1}=11.5, \epsilon_{2}=30.5, \mu_{R}=20, \mu_{L}=5$, $V_{R}=V_{L}=-50, \Gamma_{11}^{L(R)}=\Gamma_{22}^{L(R)}=\Gamma_{12}^{L}=-\Gamma_{12}^{R}=0.05, \omega=1$. As before we did not plot here the the dc - current and the first harmonic. They are are of course large compared with higher harmonics. We notice however that if $W_{R}$ becomes larger than the Fermi energy in the right lead $\left(\mu_{R}-V_{R}\right)$ they are substantially reduced, and the amplitude of the resonant harmonic might be comparable to the magnitude of the dc - current. It is clearly seen in this figure that among the high harmonics only the 19-th one $\left(n_{\text {res }}=\frac{\epsilon_{2}-\epsilon_{1}}{\omega}=19\right)$ is generated.

At $W_{L}=0$ and $\Gamma_{1} \approx \Gamma_{2}$ a simpler expression is easily obtained for the resonant harmonic:

$I_{L}^{n_{\mathrm{res}}} \approx \frac{2 e}{\hbar}\left[B_{1}\left(n_{\mathrm{res}}\right)+B_{2}\left(n_{\mathrm{res}}\right)\right] \approx i^{-n_{\mathrm{res}}} \frac{2 e}{\hbar} \frac{2 \Gamma_{12}^{L} \Gamma_{21}^{R}}{\Gamma_{1}+\Gamma_{2}} \sum_{s=\frac{\epsilon_{1}-\mu_{R}}{\omega}}^{\frac{\epsilon_{1}-V_{R}}{\omega}} J_{s}\left(\frac{W_{R}}{\omega}\right) J_{s+n_{\mathrm{res}}}\left(\frac{W_{R}}{\omega}\right)$

Only the terms with $s$ so that $\epsilon_{1}-s \omega$ is inside the right band: $V_{R}<\epsilon_{1}-s \omega<$ $\mu_{R}$ contribute to the sum. We notice that $J_{s}\left(\frac{W_{R}}{\omega}\right)$ tends to zero when $s$ becomes larger than $\frac{W_{R}}{\omega}$ if $\frac{W_{R}}{\omega} \gg 1$ so $V_{R}$ does not influence the results as soon as $V_{R}<\epsilon_{1}-W_{R}$.

In fig.6 we drew the dependence of the resonant harmonic $\left(\left|I_{L}^{n_{\text {res }}}\right|\right)$ on both $\frac{W_{R}}{\omega}$ (i.e. ac - voltage) and $\frac{\epsilon_{1}-\mu_{R}}{\omega}$ (determined by the dc - bias) for $n_{\text {res }}=\frac{\epsilon_{2}-\epsilon_{1}}{\omega}=8, \Gamma_{11}^{L(R)} / \omega=\Gamma_{22}^{L(R)} / \omega=\Gamma_{12}^{L} / \omega=-\Gamma_{21}^{R} / \omega=0.05, \mu_{R} / \omega=20$, $V_{R} / \omega=-200$. There is no generation $\left(I_{L}^{n_{\text {res }}}=0\right)$ if $W_{R}<n_{\text {res }} \omega / 2=$ $\left(\epsilon_{2}-\epsilon_{1}\right) / 2$ because the conditions $|s|<\frac{W_{R}}{\omega}$ and $\left|s+n_{\text {res }}\right|<\frac{W_{R}}{\omega}$ can not be satisfied together so one of $J_{s}\left(\frac{W_{R}}{\omega}\right), J_{s+n_{\text {res }}}\left(\frac{W_{R}}{\omega}\right)$ is small. The generation becomes significant for $W_{R}>\epsilon_{2}-\epsilon_{1}$. It is especially important if there are more than two energy levels: distant pairs of levels do not generate harmonics.

The dependence on the bias (i.e. $\frac{\epsilon_{1}-\mu_{R}}{\omega}$ ) is oscillating. The reason is the interference of different components of the wave function (remember that in a strong ac - field it is spread over a set of energies $\epsilon_{p} \pm s \omega$ with different $s)$. It shows in the formulas as oscillating behavior of $J_{s}\left(\frac{W_{R}}{\omega}\right)$ via $s$. Let us 
consider the sum in (25) at $\left|\epsilon_{1}-\mu_{R}\right| \ll W_{R}$ and $V_{R}<\epsilon_{1}-W_{R}$. It is useful to divide it into two parts:

$$
\sum_{s=s_{1}}^{\infty} J_{s}\left(\frac{W_{R}}{\omega}\right) J_{s+n_{\mathrm{res}}}\left(\frac{W_{R}}{\omega}\right)=\sum_{s=s_{1}}^{s^{*}} J_{s}\left(\frac{W_{R}}{\omega}\right) J_{s+n_{\mathrm{res}}}\left(\frac{W_{R}}{\omega}\right)+\sum_{s=s_{*}}^{\infty} J_{s}\left(\frac{W_{R}}{\omega}\right) J_{s+n_{\mathrm{res}}}\left(\frac{W_{R}}{\omega}\right)
$$

where $s_{1} \equiv \frac{\epsilon_{1}-\mu_{R}}{\omega}$ and $\left|s^{*}\right| \ll W_{R}$. The second part does not depend on $\epsilon_{1}-\mu_{R}$. The Bessel's functions in the first part can be approximated by cos:

$$
J_{s}\left(\frac{W_{R}}{\omega}\right)=\sqrt{\frac{2}{\pi \nu}} \cos \left(\nu-s \arcsin \frac{\nu}{W_{R} / \omega}-\frac{\pi}{4}\right), \nu \equiv \sqrt{\left(\frac{W_{R}}{\omega}\right)^{2}-s^{2}}
$$

since $|s| \ll \frac{W_{R}}{\omega}$. Then

$$
\begin{aligned}
I_{L}^{n_{\mathrm{res}}} & \approx i^{-n_{\mathrm{res}}} \frac{2 e}{\hbar} \frac{2 \Gamma_{12}^{L} \Gamma_{21}^{R}}{\Gamma_{1}+\Gamma_{2}} \frac{1}{\pi W_{R} / \omega} \sum_{s=s_{1}}^{s=s^{*}}\left[\cos \left(\alpha-\frac{n_{\mathrm{res}} \omega}{W_{R}} s\right)+\cos (\beta-\pi s)\right]+\text { const } \\
& \approx i^{-n_{\mathrm{res}}} \frac{2 e}{\hbar} \frac{2 \Gamma_{12}^{L} \Gamma_{21}^{R}}{\Gamma_{1}+\Gamma_{2}} \frac{1}{\pi n_{\mathrm{res}}} \sin \left(\alpha-n_{\mathrm{res}} \frac{\epsilon_{1}-\mu_{R}}{W_{R}} s\right) \quad(+\mathrm{const})
\end{aligned}
$$

where $\alpha \approx \frac{\omega n_{\mathrm{res}}^{2}}{2 W_{R}}+\frac{\pi n_{\mathrm{res}}}{2}$ and $\beta \approx 2 \frac{W_{R}}{\omega}-\frac{\pi\left(n_{\mathrm{res}}+1\right)}{2}$. When $\epsilon_{1}-\mu_{R}$ changes from $-W_{R}$ to $W_{R}$ the value $I_{L}^{n_{\text {res }}}$ oscillates about $n_{\text {res }} / \pi$ times. The amplitude of the current $\left|I_{L}^{n_{\text {res }}}\right|$ has about $2 n_{\text {res }} / \pi$ maxima (in fig.6 the number of maxima is even larger because the frequency of oscillations is larger at $\epsilon_{1}-\mu_{R} \approx$ $\left.\pm W_{R}\right)$. Notice that the current is not generated if $\left|\epsilon_{1}-\mu_{R}\right|>W_{R}$. It is significant for systems with many energy levels: only those pairs of levels that are in the energy range from $\mu_{R}-W_{R}$ to $\mu_{R}+W_{R}$ generate harmonics.

The dependence of the current on the transparency of the barriers is clear from formulas (23), (25). In fig.5 we showed the spectrum of the tunneling current at $\Gamma / \omega=1 / 20$. If $\Gamma$ increases two sets of harmonics grow in the vicinity of the 0 -th and the $n_{\text {res }}$-th harmonics. The ratio of the amplitudes of the side harmonics to the amplitude of the leading ( 0 -th or $n_{\text {res }}$-th) one is about $\Gamma / n \omega$ and $\Gamma /\left(n-n_{\text {res }}\right) \omega$ respectively. On the other hand, the magnitude of the resonant current is proportional to $\frac{2 \Gamma_{12}^{L} \Gamma_{21}^{R}}{\Gamma_{1}+\Gamma_{2}}$.

The dependence of the current on frequency is oscillatory. If a rectifying device like a diode is placed in the output the harmonics contribute to the dc - current. In fig.7 we draw the value $\langle I\rangle \equiv \frac{1}{2}\left|I_{L}^{0}\right|+\frac{2}{\pi} \sum_{n=1}^{+\infty}\left|I_{L}^{n}\right|$ coming from time - averaging of $I_{L}^{T}(t)$ as a function of the input frequency $\omega$ at 
$W_{L}=0, W_{R}=30, \epsilon_{1}=11.5, \epsilon_{2}=31.5, \mu_{R}=20, \mu_{L}=5, V_{R}=V_{L}=-200$, $\Gamma_{11}^{L(R)}=\Gamma_{22}^{L(R)}=\Gamma_{12}^{L}=-\Gamma_{12}^{R}=0.05$. The peaks correspond to different harmonics $(n=22,21,20,19,18)$ satisfying the resonant condition $n=\frac{\epsilon_{2}-\epsilon_{1}}{\omega}$.

We have discussed the high harmonic resonance in the tunneling current through the left barrier. To observe it the measuring device must be made sensitive to the tunneling current only through this barrier. Otherwise other harmonics will mask the resonance (like in fig.1). If the device measures the current in the leads we propose two ways to obtain it based on the formula (20):

1. Making the gate capacitance small: $C_{g} \ll C_{L}, C_{R}$ and the capacitance of the right barrier much larger than the capacitance of the left one: $C_{L} \ll C_{R}$. It can be achieved by making the left barrier thicker than the right one. Notice that the right barrier must be made slightly higher so that $\Gamma_{L}$ and $\Gamma_{R}$ are of the same order (then $I_{L}^{T}$ and $I_{R}^{T}$ are of the same order). Thus the high harmonic currents in the leads are approximately given by:

$$
\begin{aligned}
& I_{L}(t) \approx I_{L}^{T}(t)-\frac{C_{L}}{C_{R}} I_{R}^{T}(t) \approx I_{L}^{T}(t) \\
& I_{R}(t) \approx-I_{L}^{T}(t)+\frac{C_{L}}{C_{R}} I_{R}^{T}(t) \approx-I_{L}^{T}(t)
\end{aligned}
$$

We omitted here the contribution of the capacitive currents having frequency $\omega$. The high harmonic currents in both leads are determined by $I_{L}^{T}$.

2. Making the gate capacitance large: $C_{g} \gg C_{L}$ (the ratio of barrier capacitances $C_{L}$ and $C_{R}$ is arbitrary, there is no need to make one of the barriers higher than the other one). Then (dropping out the capacitive currents) we obtain from (20):

$$
\begin{aligned}
I_{L}(t) & \approx I_{L}^{T}(t)-\frac{C_{L}}{C_{R}+C_{g}} I_{R}^{T}(t) \\
I_{R}(t) & \approx-\frac{C_{R}}{C_{R}+C_{g}} I_{L}^{T}(t)+\frac{C_{g}}{C_{R}+C_{g}} I_{R}^{T}(t)
\end{aligned}
$$

We notice that in this case high harmonics in the left lead are produced by $I_{L}^{T}$ but in the right lead by both $I_{L}^{T}$ and $I_{R}^{T}$ (unless $C_{R} \gg C_{g}$ ). If 
$W_{L}=0, W_{R} \neq 0$ the term $A$ generating a wide spectrum of harmonics vanishes in $I_{L}^{T}(t)$ but it is not zero in the expression for $I_{R}^{T}(t)$. Then the current in the right lead $I_{R}(t)$ consists of many harmonics. To detect a single harmonic the current in the left lead should be measured.

\section{Summary}

The basic physical problem addressed in this work is concerned with a nonlinear response of a two(or more)-level system to a high frequency external field. This makes the formalism developed above particularly attractive since two-level systems are an important model for many realistic physical situations. The most familiar one is evidently a two-level atomic system, whose response to a laser field is one of the hall marks of non-linear quantum optics, one of whose signatures is higher frequency generation. Here we have focused on an electronic analog, where the response is a tunneling current instead of an emitted light. Note that, unlike the optical analog, the theoretical formulation requires computation of non-equilibrium (Schwinger-Keldysh) Green functions. As far as the experimental situation is concerned, we see no real obstacle in the road for actual observation of this effect.

We expect new effects to occur when the many-body physics is included. When the size of the dot is small enough then, at voltages and frequencies less then the Coulomb interaction energy (which is frequently the case), it can be considered as an Anderson impurity, which shows a Kondo type effect if the resonance level is deep below the Fermi level and the temperature is low enough. Hence, the first problem which comes into mind in this context is a non-linear response of a magnetic impurity to a time-dependent field. The Kondo effect out of equilibrium has been studied recently by several authors, but this is done primarily in the non-crossing approximation, which is valid much above the Kondo temperature. The formalism developed above gives the hope that also crossed diagrams can be included (the first ones appear when a sixth order term in the tunneling matrix elements are computed) and therefore it can approach the physics at temperatures lower than the Kondo temperature. If external voltages or frequencies are comparable with the Coulomb interaction energy mutual time-dependent resonant tunneling of electrons might show new physics. We suppose that this problem can be handled using the finite - $U$ Anderson model. 
We would like to thank A. Golub, J. Golub and N. S. Wingreen for very helpful discussion. The research of Y. A is partially supported by the Fund for Basic Research of the Israeli Academy of Science and by the Israeli Ministry of Science and the Arts.

\section{Appendix A}

In this Appendix we show that an arbitrary strong time - dependent potential has no effect if it is uniform in space. We also calculate here Green functions for an isolated lead.

If the alternating potential $\left(H_{a c}(t)\right)$ is uniform the Hamiltonian can be written in the following form:

$$
H(t)=H_{f}+H_{a c}(t)
$$

where $H_{f}$ is time - independent, $H_{a c}(t)$ is space - independent. The solutions of the Schrödinger's equation are:

$$
\Psi_{k}(t)=\varphi_{k}(t) e^{-\frac{i}{\hbar} \int^{t} H_{a c}\left(t_{1}\right) d t_{1}}
$$

where $\varphi_{k}(t)$ are eigenstates of $H_{f}$. The time dependent part $H_{a c}(t)$ influences only the phases giving all the solutions the same phase factor $e^{-\frac{i}{\hbar} \int^{t} H_{a c}\left(t_{1}\right) d t_{1}}$. The phase differences are determined by $H_{f}$ only. Thus $H_{a c}(t)$ has no physical effect. This simple result is the time - dependent analog of the fact that the (time - independent) reference point of energy can be arbitrarily chosen.

We used this result to write the ac - part of the Hamiltonian of a double - barrier structure in the form (4). The ac - shift of the central region is ignored.

In an isolated lead the ac - potential is uniform (see (田). Then the evolution operator $\left(U\left(t, t^{\prime}\right)\right)$ is obtained from the equation $i \hbar \frac{\partial}{\partial t} U\left(t, t^{\prime}\right)=$ $H(t) U\left(t, t^{\prime}\right)$ with $H(t)$ given by (27). Its matrix elements are:

$$
U_{k k^{\prime}}\left(t, t^{\prime}\right)=\delta_{k k^{\prime}} e^{-\frac{i}{\hbar} \int_{t^{\prime}}^{t} H_{k k}\left(t_{1}\right) d t_{1}}
$$

$\delta_{k k^{\prime}}$ appears because $H_{k k^{\prime}}=0, k \neq k^{\prime}$ (i.e a uniform potential does not cause transitions). Using the definition of Green functions in the Schrödinger representation with the evolution operator (29) it is easy to obtain the formulas (13). 


\section{Appendix B}

In this Appendix we analyze the Dyson's equation for $G_{n m}^{<}$and obtain the formula (11). The Dyson's equation (7) holds for $G_{n m}^{<}$:

$$
\begin{aligned}
G_{n m}^{<} & =F_{n m}^{1}\left(g_{j j}^{<}\right)+F_{n m}^{2}\left(g_{k k}^{<}, g_{p p}^{<}\right) \\
F_{n m}^{1}\left(g_{j j}^{<}\right) & \equiv g_{n m}^{<}+G_{n k}^{r} T_{k m} g_{m m}^{<}+G_{n p}^{r} T_{p m} g_{m m}^{<}+ \\
& +g_{n n}^{<} T_{n k} G_{k m}^{a}+g_{n n}^{<} T_{n p} G_{p m}^{a}+ \\
& +G_{n k}^{r} T_{k i} g_{i i}^{<} T_{i k^{\prime}} G_{k^{\prime} m}^{a}+G_{n p}^{r} T_{p i} g_{i i}^{<} T_{i p^{\prime}} G_{p^{\prime} m}^{a}+ \\
& +G_{n k}^{r} T_{k i} g_{i i}^{<} T_{i p} G_{p m}^{a}+G_{n p}^{r} T_{p i} g_{i i}^{<} T_{i k} G_{k m}^{a} \\
F_{n m}^{2}\left(g_{k k}^{<}, g_{p p}^{<}\right) & \equiv G_{n n^{\prime}}^{r} T_{n^{\prime} k} g_{k k}^{<} T_{k m^{\prime}} G_{m^{\prime} m}^{a}+G_{n n^{\prime}}^{r} T_{n^{\prime} p} g_{p p}^{<} T_{p m^{\prime}} G_{m^{\prime} m}^{a}
\end{aligned}
$$

Here multiplication implies integration over time and summation over repeated indexes; $j=m, n, i$ belong to the central region, $k, k^{\prime}$ — to the left lead, $p, p^{\prime}$ — to the right lead.

$F_{n m}^{1}\left(g_{j j}^{<}\right)$depends on the initial state of the central region. It does not depend on $g_{k k}^{<}, g_{p p}^{<}\left(G^{r}, G^{a}\right.$ are determined by the equation (6) which does not depend on $\left.g_{k k}^{<}, g_{p p}^{<}\right)$. It describes the behavior of the system with empty leads. Indeed, if $g_{k k}^{<}=0, g_{p p}^{<}=0$ we have $F_{n m}^{2}=0$. Then $G_{n m}^{<}=F_{n m}^{1}$. When the leads are empty the central region empties with time. $F_{n m}^{1}$ tends to zero. After a long enough period of time has passed from the moment the tunneling was switched on it can be neglected. Then $G_{n m}^{<}=F_{n m}^{2}$. This is the formula (11). We can say that $F_{n m}^{1}$ describes the transient processes while $F_{n m}^{2}$ gives some kind of a quasistationary (but fully time - dependent) solution.

\section{References}

[1] J. B. Pieper and J. C. Price, Phys. Rev. Lett. 72, 3586 (1994).

[2] M.Bü ttiker, A.Prêtre, H.Thomas, Phys.Rev.Lett., 70, 4114 (June 1993).

[3] D. Z. Liu, B. Y. K. Hu, C. A. Stafford and S. Das Sarma, Phys. Rev. B50, 5799 (1994). 
[4] J. B. Pieper and J. C. Price, Phys. Rev. B49, 17059 (1994).

[5] L. J. Greelings et al., Phys. Rev. Lett. 64, 2691 (1990).

[6] L.P.Kouwenhoven, A.T.Johnson, N.C.van der Vaart, C.J.P.M.Harmans, C.T.Foxon, Phys.Rev.Lett., 67, 1626 (1991).

[7] C.A.Stafford, N.D.Wingreen, Resonant Photon-Assisted Tunneling Through a Quantum Dot: An Electron Pump from Spatial Rabi Oscillations, preprint VGVA-DPT 1995/09-901

[8] J. Faist et al., Science, 264, 553 (1994).

[9] R.K.Tien, J.P.Gordon, Phys.Rev., 129, 647 (1963).

[10] L.P.Kouwenhoven, S.Jauhar, K.McCormick, D.Dixon, P.L.McEuen, Yu.V.Nazarov, N.C.van der Vaart, C.T.Foxon, Phys.Rev.B, 50, 2019 (July 1994).

[11] L.P.Kouwenhoven, S.Jauhar, J.Orenstein, P.L.McEuen, Y.Nagamune, J.Motohisa, H.Sakaki, Phys.Rev.Lett., 73, 3443 (Dec. 1994).

[12] C.Bruder, H.Schoeller, Phys.Rev.Lett., 72, 1076 (Feb. 1994).

[13] H. Akiyama et al., Appl. Phys. Lett. 65, 424 (1994).

[14] R. H. Blick, R. J. Haug, K. von Klitzing and K. Eberl, preprint.

[15] L.L.Chang, L.Esaki, R.Tsu, Appl.Phys.Lett., 24, 593 (June 1974).

[16] T.C.L.G.Sollner, W.D.Goodhue, P.E.Tannenwald, C.D.Parker, D.D.Peck, Appl.Phys.Lett., 43, 588 (Sept. 1983).

[17] T.C.L.G.Sollner, P.E.Tannenwald, D.D.Peck, W.D.Goodhue, Appl.Phys.Lett., 45, 1319 (Dec. 1984). 
[18] J.F.Whitaker, G.A.Mourou, T.C.L.G.Sollner, W.D.Goodhue, Appl.Phys.Lett., 53, 385 (Aug. 1988).

[19] V.J.Goldman, D.C.Tsui, J.E.Cunningham, Phys.Rev.Lett., 58, 1256 (March 1987).

[20] V.J.Goldman, D.C.Tsui, J.E.Cunningham, Phys.Rev.B, 35, 9387 (June 1987).

[21] M.A.Reed, J.N.Randall, R.J.Aggarwal, R.J.Matyi, T.M.Moore, A.E.Wetsel, Phys.Rev.Lett., 60, 535 (Feb. 1988).

[22] A.Rydberg, H.Grönqvist, Electr.Lett., 25, 348 (March 1989).

[23] E.R.Brown, J.R.Söderström, C.D.Parker, L.J.Mahoney, K.M.Molvar, T.C.McGill, Appl.Phys.Lett., 58, 2291 (May 1991).

[24] Bo Su, V.J.Goldman, J.E.Cunningham, Science, v.255, p.313, Jan. 1992.

[25] M.W.Dellow, P.H.Beton, C.J.G.M.Langerak, T.J.Foster, P.C.Main, L.Eaves, M.Henini, S.P.Beaumont, C.D.W.Wilkinson, Phys.Rev.Lett., 68, 1754 (Mar. 1992).

[26] P.Guéret, N.Blanc, R.Germann, .Rothuizen, Phys.Rev.Lett., 68, 1896 (Mar. 1992).

[27] R.C.Ashoori, H.L.Stormer, J.S.Weiner, L.N.Pfeiffer, S.J.Pearton, K.W.Baldwin, K.W.West, Phys.Rev.Lett., 68, 3088 (May 1992).

[28] U.Meirav, M.A.Kastner, S.J.Wind, Phys.Rev.Lett., 65, 771 (Aug. 1990).

[29] P.L.McEuen, E.B.Foxman, U.Meirav, M.Kastner, Y.Meir, N.S.Wingreen, S.J.Wind, Phys.Rev.Lett., 66, 1926 (Apr. 1991).

[30] C.J.B.Ford, P.J.Simpson, M.Pepper, D.Kern, J.E.F.Frost, D.A.Ritchie, G.A.C.Jones, 
[31] M.Field, C.G.Smith, M.Pepper, D.A.Ritchie, J.E.F.Frost, G.A.C.Jones, D.G.Hasko, Phys.Rev.Lett., 70, 1311 (Mar. 1993).

[32] E.B.Foxman, P.L.McEuen, U.Meirav, N.S.Wingreen, Y.Meir, P.A.Belk, N.R.Belk, M.Kastner, Phys.Rev.B, 47, 10020 (Apr. 1993).

[33] J.Weis, R.J.Haug, K.v.Klitzing, K.Ploog, Phys.Rev.Lett., 71, 4019 (Dec. 1993).

[34] B.Meurer, D.Heitmann, K.Ploog, Phys.Rev.Lett., 68, 1371 (Mar. 1992).

[35] A.T.Johnson, L.P.Kouwenhoven, W.de Jong, N.C.van der Vaart, C.J.P.M.Harmans, C.T.Foxon, Phys.Rev.Lett., 69, 1592 (Sept. 1992).

[36] K.Nakazato, T.J.Thornton, J.White, H.Ahmed, Appl.Phys.Lett., 61, 3145 (Dec. 1992).

[37] A.Yacoby, M.Heiblum, D.Mahalu, H.Shtrikman, Phys.Rev.Lett., 74, 4047 (May 1995).

[38] A.B.Fowler, G.L.Timp, J.J.Wainer, R.A.Webb, Phys.Rev.Lett., 57, 138 (July 1986).

[39] T.E.Kopley, P.L.McEuen, R.G.Wheeler, Phys.Rev.Lett., 61, 1654 (Oct. 1988).

[40] L.W.Molenkamp, K.Flensberg, M.Kemerink,

[41] D.C.Ralph, R.A.Buhrman, Phys.Rev.Lett., 72, 3401 (May 1994).

[42] J.Bardeen, Phys.Rev.Lett., 6, 2 (Jan. 1961).

[43] M.H.Cohen, L.M.Falicov, J.C.Phillips, Phys.Rev.Lett., 8, 316 (Apr. 1962).

[44] L.Y.Chen, C.S.Ting, Phys.Rev.B, 43, 2097 (1991). 
[45] W.R.Frensley, Superlattices and Microstructures, 4, 497 (1988).

[46] Y.Meir, N.S.Wingreen, Phys.Rev.Lett., 68, 2512 (Apr. 1992).

[47] A.Sugimura, Phys.Rev.B, 47, 9676 (Apr. 1993).

[48] J.R.Tucker, IEEE J.Quant.Elec., QE-15, 1234 (1979).

[49] P.Johansson, Phys.Rev.B, 41, 9892 (1990).

[50] H.C.Liu, Phys.Rev.B, 43, 12538 (1991).

[51] W.Cai, P.Hu, M.Lax, Phys.Rev.B, 44, 3336 (1991).

[52] Z.-B.Su, Lu Yu, L.-Y.Chen, in: Thermal Field Theories, eds. H.Egawa, T.Arimitru, Y.Hashimoto, p.107, (Elsevier, 1991).

[53] Y.Isawa,in Transport Phenomena in Mesoscopic Systems, ed. by H.Fukuyama and T.Ando, Springer Series in Solid-State Sciences, v.109, p.93, (Springer, Berlin, 1992).

[54] R.Berkovits, M.Abraham, Y.Avishai, J.Phys.: Condensed. Matter, 5, L175 (1993).

[55] N.S.Wingreen, A.P.Jauho, Y.Meir, Phys.Rev.B, 48, 8487 (Sept. 1993).

[56] L.Y.Chen, C.S.Ting, Phys.Rev.Lett., 64, 3159 (1990).

[57] B.Ricco, M.Ya.Azbel, Phys.Rev.B, 29, 1970 (Feb. 1984).

[58] A.D.Stone, M.Ya.Azbel, P.A.Lee, Phys.Rev.B, 31, 1707 (1985).

[59] T.Weil, B.Vinter, Appl.Phys.Lett., 50, 1281 (May 1987).

[60] M.Jonson, A.Grincwaig, Appl.Phys.Lett., 51, 1729 (Nov. 1987).

[61] M.Büttiker, IBM J.Res.Develop., 32, 63 (Jan. 1988). 
[62] D.Sokolovski, J.Phys.C: Solid State Phys., 21, 639 (1988).

[63] M.Yu.Sumetskii, M.L.Fel'shtyn, Sov.Phys.JETP, 67, 1610 (1988).

[64] P.Price, Phys.Rev.B, 43, 2097 (Jan. 1991).

[65] F.W.Sheard, G.A.Toombs, Appl.Phys.Lett., 52, 1228 (Apr. 1988).

[66] D.V.Averin, A.N.Korotkov, K.K.Likharev, Phys.Rev.B, 44, 6199 (Sept. 1991).

[67] T.Figg, A.P.Jauho, Surf.Sci., 267, 392 (1992).

[68] J.Schwinger, J.Math.Phys., 2, 407 (1961).

[69] L.P.Kadanoff, G.Baym, Quantum Statistical Mechanics, 203p., W.A.Benjamin Inc., N.Y., 1962.

[70] L.V.Keldysh, Sov.Phys.JETP, 20, 1018 (1965).

[71] L.D.Landau, E.M.Lifshitz, Course of Theoretical Physics, v.10, Physical Kinetics, p.395, (Pergamon Press, 1981).

[72] K.-C.Chou, Z.-B.Su, B.-L.Hao, Lu Yu, Phys.Rep. (Rev. Section. Phys.Lett), 118, 1 (1985).

[73] G.D.Mahan, Many-Particle Physics, 1990.

[74] G.D.Mahan, Phys.Rep. (Rev. Section. Phys.Lett), 145, 251 (1987).

[75] J.Rammer, H.Smith, Rev.Mod.Phys., 58, 323 (1986).

[76] C.Caroli, R.Combescot, P.Nozieres, D.Saint-James, J.Phys.C: Solid State Phys., 4, 916 (1971).

[77] N.S.Wingreen, K.W.Jacobsen, J.W.Wilkins, Phys.Rev.B, 40, 11834 (Dec. 1989). 
[78] D.C.Langreth, P.Nordlander, Phys.Rev.B, 43, 2541 (Feb. 1991).

[79] G.-L.Ingold, Yu.V.Nazarov, in: Single Charge Tunneling, ed. by H.Grabert, M.H.Devoret, N.Y., 1992. 
Figure 1: Schematic energy diagram of the conduction band for an $A l_{x} G a_{1-x} A s$ quantum well in the absence of an ac-field. $x$ is the axis perpendicular to the layers, $\mu_{L}$ and $\mu_{R}$ are the left and the right chemical potentials correspondently, $V_{L}$ and $V_{R}$ are the energies of the conduction band bottoms of the leads, $\epsilon_{1}$ and $\epsilon_{2}$ are the energies of the levels in the central region.

Figure 2: Tunneling diagrams. A cross denotes a tunneling event, a thing line - free propagator, a thick line - full propagator. a) Dyson's equation. Only a single tunneling event contributes to the self - energy. b) any other diagram is reducible.

Figure 3: Tunneling processes leading to the high harmonic resonance. A thick black line denotes full electron propagator, a thick white one - full hole propagator, a thing black line - free electron propagator, a dashed line - tunneling event. a) The tunneling current is produced by electrons going from the lead to a level in the central region and back. b) This process can be assisted by other levels if ac - field is strong. c) Resonance transfer of an electron from the level $m$ to the level $n$ possible in a strong ac-field.

Figure 4: Spectrum of the tunneling current (amplitude of the harmonics via their number). General situation. $W_{L}=W_{R}=30, \epsilon_{1}=11.5, \epsilon_{2}=30.5$, $\mu_{R}=20, \mu_{L}=5, V_{R}=V_{L}=-50, \Gamma \equiv \Gamma_{11}^{L(R)}=\Gamma_{22}^{L(R)}=\Gamma_{12}^{L}=-\Gamma_{12}^{R}=0.05$, $\omega=1$. The dc - current and the 1-st harmonic are not shown.

Figure 5: Spectrum of the tunneling current (amplitude of the harmonics via their number) when the alternating field is applied on the right barrier: $W_{L}=0, W_{R}=30, \epsilon_{1}=11.5, \epsilon_{2}=30.5, \mu_{R}=20, \mu_{L}=5, V_{R}=V_{L}=-50$, $\Gamma \equiv \Gamma_{11}^{L(R)}=\Gamma_{22}^{L(R)}=\Gamma_{12}^{L}=-\Gamma_{12}^{R}=0.05, \omega=1$. The dc - current and the 1-st harmonic are not shown.

Figure 6: Dependence of the resonant harmonic $\left(\left|I_{L}^{n_{\text {res }}}\right|\right)$ on the dc - bias (i.e. $\epsilon_{1}-\mu_{R}$ ) and the amplitude of the ac - voltage $W_{R} \cdot n_{\text {res }}=8, W_{L}=0$, $\epsilon_{2}=\epsilon_{1}+8, \mu_{R}=20, V_{R}=-200, \Gamma_{11}^{L(R)}=\Gamma_{22}^{L(R)}=\Gamma_{12}^{L}=-\Gamma_{12}^{R}=0.05, \omega=1$.

Figure 7: The output current rectified by a diode versus the frequency $\omega$ of the input ac - voltage. $W_{L}=0, W_{R}=30, \epsilon_{1}=11.5, \epsilon_{2}=31.5, \mu_{R}=20$, $\mu_{L}=5, V_{R}=V_{L}=-200, \Gamma \equiv \Gamma_{11}^{L(R)}=\Gamma_{22}^{L(R)}=\Gamma_{12}^{L}=-\Gamma_{12}^{R}=0.05$. The peaks correspond to different harmonics $(n=22,21,20,19,18)$ satisfying the resonant condition $n=\frac{\epsilon_{2}-\epsilon_{1}}{\omega}$. 